\title{
La evaluación formativa en un contexto de renovación pedagógica: Prácticas al servicio del éxito
}

Formative Evaluation in a Context of Pedagogical Renewal:

Practices at the service of success

\section{Volumen 20, Número 1 \\ Enero - Abril \\ pp. 1-26}

\author{
Silvia Verónica Valdivia Yábar \\ María Estela del Carmen Fernández Guillén
}

\section{Citar este documento según modelo APA}

Valdivia Yábar, Silvia Verónica y Fernández Guillén, María Estela del Carmen. (2020). La evaluación formativa en un contexto de renovación pedagógica: Prácticas al servicio del éxito. Revista Actualidades Investigativas en Educación, 20(1), 1-26. Doi. 10.15517/aie.v20i1.40159 


\title{
La evaluación formativa en un contexto de renovación pedagógica: Prácticas al servicio del éxito \\ Formative Evaluation in a Context of Pedagogical Renewal: Practices at the service of success
}

\section{Silvia Verónica Valdivia Yábar ${ }^{1}$ María Estela del Carmen Fernández Guillén²}

\begin{abstract}
Resumen: En el contexto de renovación pedagógica peruana la apropiación por el profesorado de los cambios prescritos en el plan de evaluación necesita de la difusión de prácticas ejemplares, puesto que varias investigaciones rigurosas muestran que la observación de ejemplos puede favorecer el aprendizaje de nuevas prácticas de enseñanza. Por tanto, el objetivo principal de la investigación, que se realizó el segundo semestre del año 2017, y se reporta en este artículo, fue caracterizar las prácticas de evaluación formativa del personal docente de educación primaria. Se aplicó el enfoque cualitativo y se adoptaron los diseños tanto etnográfico como estudios de casos. Se recopilaron los datos, primero, acerca de la planificación de la actividad con una entrevista a 13 docentes que participaron voluntariamente; luego, sobre las acciones del profesorado y del estudiantado mediante la observación filmada de 25 actividades de aprendizaje-enseñanza; finalmente, acerca de los segmentos significativos de la actividad grabada con una entrevista al profesorado. Las prácticas espontáneas del personal docente fueron analizadas en su doble dimensión conductual y cognitiva. La investigación se centró en los diferentes elementos de los procesos requeridos y de los dispositivos implementados, así como en los contenidos tratados. Los resultados muestran que el personal docente integró a sus prácticas varias características de la evaluación formativa recomendada por el Ministerio de Educación del Perú y que su principal desafío consiste en compartir la responsabilidad de los procesos de regulación con el estudiantado mediante la creación de las condiciones para desarrollar su capacidad de autorregular sus aprendizajes.
\end{abstract}

Palabras clave: evaluación formativa, práctica de la enseñanza, desarrollo profesional

\begin{abstract}
In the context of the Peruvian pedagogical renewal, the appropriation by teachers of the prescribed changes in the evaluation plan requires the dissemination of exemplary practices, since several rigorous investigations show that observing examples can favor the learning of new teaching practices. Therefore, the main objective of this research, which was carried out during the second half of 2017, and is reported in this article, has been to characterize the formative evaluation practices of primary school teachers. The qualitative approach was applied and both ethnographic and case study designs were adopted. Data was collected, first, about the planning of the activity with an interview with13 volunteer teachers; then, on the actions of teachers and students through the filmed observation of 25 learning-teaching activities; finally, about the significant segments of the activity recorded through an interview with the teachers. The spontaneous practices of teachers have been analyzed in their double behavioral and cognitive dimension. The study focused on the different elements of the required processes and the devices implemented; as well as, about the contents treated. The results show that the participants have integrated into their practices many characteristics of the formative evaluation, recommended by the Ministry of Education, and that their main challenge consists in sharing the responsibility of the regulation processes with the student by creating the conditions to develop their ability to self-regulate their learning.
\end{abstract}

Key words: formative evaluation, teaching practices, professional development

\footnotetext{
${ }_{1}$ Profesora e Investigadora en la Universidad Nacional del Altiplano, Perú. Doctora en Pedagogía de la Universidad Nacional Autónoma de México. México. Dirección electrónica: siveroval@gmail.com ORCID https://orcid.org/0000-0002-9665-5022

1 Profesora de la Universidad Nacional Autónoma de México. México. Maestra en Pedagogía de la Universidad Nacional Autónoma de México. México. Dirección electrónica: estelafernandez49@yahoo.com.mx ORCID https://orcid.org/0000-0003$\underline{4409-4881}$
}

Artículo recibido: 19 de junio, 2019

Enviado a corrección: 17 de setiembre, 2019

Aprobado: 18 de noviembre, 2019 


\section{Introducción}

Desde hace unos años en el Perú, la cuestión del éxito escolar está cada vez más en el centro de los debates sociales. El currículo de formación de la educación básica peruana asocia el éxito escolar a la misión de calidad de la escuela (Ministerio de Educación del Perú, 2016). Hadji (1999) consideró que la evaluación se debe convertir en una poderosa palanca para aumentar el éxito en la escuela. Para este propósito, la mayor parte de las investigaciones sobre la evaluación del aprendizaje sostiene que es necesario romper con las prácticas que tienden a situar la evaluación al final del aprendizaje (Rosales, 2003). De allí, la importancia de una evaluación formativa, concomitante con el aprendizaje. La revisión de la investigación sobre la evaluación en el aula de Black y Wiliam (1998), que marcó un hito, descubrió que los esfuerzos orientados a mejorar la evaluación formativa producían beneficios mayores a la mitad de una desviación estándar. En otras palabras, la evaluación formativa, eficazmente implementada, puede hacer tanto o más para mejorar la realización y los logros que cualquier otra de las intervenciones más poderosas de la enseñanza, tales como la enseñanza intensiva de lectura, las clases particulares y otras parecidas.

Por su parte, la investigación de Solé (2001) muestra que, en lectura, el estudiantado expuesto a las prácticas de evaluación, centradas en el apoyo al aprendizaje y los progresos individuales, tiende a estar más orientado hacia el aprendizaje que el alumnado expuesto a las prácticas evaluativas, centradas en el desempeño. El mismo estudio indica que, en el estudiantado, una orientación hacia el aprendizaje está relacionada positivamente con todos los componentes del concepto de sí mismo (escolar o no), componentes relacionados con los resultados escolares.

En estudios de casos de dos localidades, australiana e inglesa, Klenowski (1995) muestra la relación entre una estrategia de autoevaluación implementada por el estudiantado y el control que éste ejerce sobre sus aprendizajes. Los datos de Klenowski (1995) respaldan la aseveración previa de Wiggins (1992) de que hacer que el estudiantado analice su propio trabajo hace que se apropie del proceso de evaluación y "hace posible llevar a los estudiantes a niveles más altos porque los criterios son claros y razonables" (p. 30). En un contexto de trabajo colaborativo, la investigación de Cohen, Lotan, Abram, Scarloss y Schultz (2002) indica el efecto directo de una estrategia que consiste en comunicar los criterios de evaluación al grupo de trabajo sobre la naturaleza del grupo y sobre el desempeño del mismo. 
Apoyándose en estos hallazgos y en el Currículo Nacional de la Educación Básica, el Ministerio de Educación del Perú (2016) exigió al personal docente modificar sus prácticas de evaluación para poner énfasis en la evaluación durante el aprendizaje. Dicha prescripción tuvo implicaciones importantes para la formación inicial y continua del profesorado, puesto que, en el momento en que se propuso la reforma curricular, prevalecía una evaluación sumativa con interpretación basada en criterios, en las prácticas evaluativas, y cuando se realizaban las evaluaciones durante el aprendizaje, la función formativa estaba ausente. Picaroni (2009) refiere que, en forma implícita o explícita, la mayoría del profesorado peruano entrevistado hace al menos alguna alusión a las funciones básicas de la evaluación, distingue entre evaluación sumativa y evaluación formativa. Sin embargo, la minoría del personal docente da cuenta de llevar adelante las acciones que esta última debería priorizar: proporcionar al estudiantado insumos concretos, claramente entendibles, para que tome conciencia de sus logros y sus errores, así como de los recorridos posibles para superarlos.

Respecto de la autoevaluación del estudiantado, la Organización para la Cooperación y el Desarrollo Económico (2014), en TALIS 2013, informó que es un método utilizado con menos frecuencia en la práctica docente: el 35,1\% del profesorado en la media OCDE informa que permite al estudiantado evaluar su propio progreso, y poco más de tres de cada cinco en Chile (68\%) dice utilizar con frecuencia este método para evaluar al alumnado. Sería muy conveniente fomentar este tipo de evaluación en el Perú, ya que favorece el análisis y la reflexión sobre el proceso de enseñanza-aprendizaje por parte del estudiantado y del profesorado, lo cual se puede traducir en una mejora del rendimiento global del alumnado.

Como enfatizan Wilson y Berne (1999), los cambios prescritos por un currículo y las directivas no transforman automáticamente las prácticas. Algunos autores, entre ellos Fletcher, Foorman, Denton y Vaughn (2006) consideran la falta de oportunidades del personal docente para observar las prácticas, que se deben implementar, como un obstáculo para cambiar. Windschitl (2002), quien se ocupa de las dificultades de adoptar algunas prácticas de enseñanza, argumenta que el profesorado necesita conocer la historia de los pares que lograron implementar nuevas prácticas. El estudio de Garet, Porter, Desimone, Birman y Yoon (2001) muestra que la participación en las actividades de aprendizaje, incluyendo la posibilidad de observar las prácticas de enseñanza expertas, constituye una de las características de los programas de formación continua con efectos positivos. 
Con relación a las iniciativas de apoyo a la cooperación entre el profesorado, según las encuestas de TALIS, una media del $64 \%$ del personal directivo señala adoptar estas ideas, con miras a desarrollar nuevas técnicas de enseñanza y evaluación. En chile, Malasia y Rumania, entre el $80 \%$ y el $98 \%$ del personal directivo de centros educativos responde apoyar asiduamente la cooperación entre sus docentes para desarrollar nuevas prácticas, mientras que en Dinamarca, Estonia y Japón más del 50\% del personal directivo señala que nunca, raramente u ocasionalmente lo hace (OCDE, 2014). El personal líder escolar peruano puede ofrecer la oportunidad al profesorado de participar en actividades de desarrollo profesional relacionadas con la mejora de sus prácticas docentes. Estas actividades deben comprender la colaboración y tutela en el propio centro educativo (Picaroni, 2009).

En síntesis, la renovación pedagógica introducida en el Perú priorizó las prácticas de evaluación formativa, lo cual representó un cambio para el personal docente. Puesto que diversas investigaciones rigurosas muestran que la observación de ejemplos puede favorecer el aprendizaje de nuevas prácticas de enseñanza, el objetivo de esta investigación ha sido caracterizar las prácticas de evaluación formativa de las profesoras y los profesores de primaria, quienes incorporaron las características de la evaluación prescritas por el Ministerio de Educación del Perú. Se utilizó el enfoque cualitativo y los diseños tanto etnográfico como estudios de casos. Esta investigación se realizó en los ciclos III, IV y V de la educación primaria en la institución educativa Corazón de Jesús de la ciudad de Puno Perú, durante el segundo semestre del año 2017.

En este artículo, primero, se definen los conceptos de práctica de enseñanza y de evaluación formativa. Luego, se explica la metodología abordada, la cual comprendió herramientas cualitativas para la obtención de los datos necesarios para el análisis y la construcción del conocimiento en el área. Seguidamente, se presentan los resultados de la investigación a través de una visión general y otra centrada en los ejemplos extraídos de tres casos. Para concluir, se identifican algunas pistas relacionadas con la formación continua.

\section{Referente teórico}

Esta investigación de las prácticas de evaluación formativa está en línea con las investigaciones sobre las prácticas de enseñanza, las cuales permiten una mejor comprensión del trabajo docente. Ésta pretende proponer un marco para aclarar la relación entre la evaluación formativa y la regulación del aprendizaje. 


\subsection{Las prácticas de enseñanza}

Al desarrollar un estado de evolución de los estudios sobre el análisis del trabajo docente, Altet (2014) informó que, al inicio, una corriente conductista se centró en las acciones observables del profesorado; luego, otra corriente llamada proceso-producto orientó el trabajo hacia el efecto de las prácticas de enseñanza en el aprendizaje del estudiantado. En seguida, la influencia de los modelos cognitivistas concentró el interés en los procesos cognitivos subyacentes en el comportamiento del personal docente (pensamiento del profesorado) (Kagan, 1990), vistos estos como factores exclusivos que controlan las prácticas. Casalfiore (2000), al respecto, destaca una corriente fenomenológica que insiste en que las metáforas, las imágenes personales y circunstanciales producidas por los profesores y las profesoras funcionan como los organizadores cognitivos de la actividad. Finalmente, los modelos ecológicos e interaccionistas reintrodujeron las variables de situación y contexto en el estudio de las prácticas.

Según lo propuesto por Paquay (2004), aunque estas corrientes se han visto en el pasado como algo exclusivo, en particular, las corrientes conductista y cognitivista, se deben considerar a la vez las dimensiones conductual y cognitiva de la acción docente, teniendo en cuenta el contexto. Aunado a ello, cada vez más, las investigaciones definen las prácticas de enseñanza como una acción situada, que Altet (2002) inscribe en un marco constructivista e interaccionista.

Una encuesta de diferentes definiciones del concepto de práctica docente (Altet, 2002) indica que estas suelen tener en cuenta una dimensión conductual (procesos de implementación observables de la actividad) y una dimensión cognitiva (las elecciones y la toma de decisiones). Estas acciones conciernen a una persona singular en una situación dada.

Las investigaciones sobre las prácticas docentes nos llevan a privilegiar el concepto de prácticas de enseñanza, un concepto menos englobante. Las prácticas docentes incluyen, por una parte, las prácticas de enseñanza y, por otra parte, otras prácticas efectuadas fuera del horario escolar, en ausencia del alumnado, incluyendo las reuniones de colegas en equipos y las reuniones con los padres de familia.

Así, inspirándose en Beillerot y Mosconi (2014), el concepto de prácticas de enseñanza, cuya finalidad es el aprendizaje del estudiantado, se define en esta investigación como el conjunto de los actos de cada profesional, observables o no, así como los significados que ella o él les otorga. Las prácticas de enseñanza incluyen entonces una 
dimensión conductual (actos observables) y otra cognitiva (actos mentales) (Figura 1). En cuanto a los significados otorgados a estos actos por cada docente, están directamente relacionados con la acción situada contrariamente a las concepciones, que son generales. Estos actos se implementan en presencia o ausencia del estudiantado, durante el horario escolar y fuera del horario escolar, individual o colectivamente (con los pares u otras personas). Finalmente, las prácticas de enseñanza incluyen las acciones realizadas en las fases preactiva (planificación de la acción), interactiva (acción en presencia del alumnado) y postactiva (evaluación de la acción) de la intervención.

Figura 1

Prácticas docentes, prácticas de enseñanza y prácticas de evaluación formativa

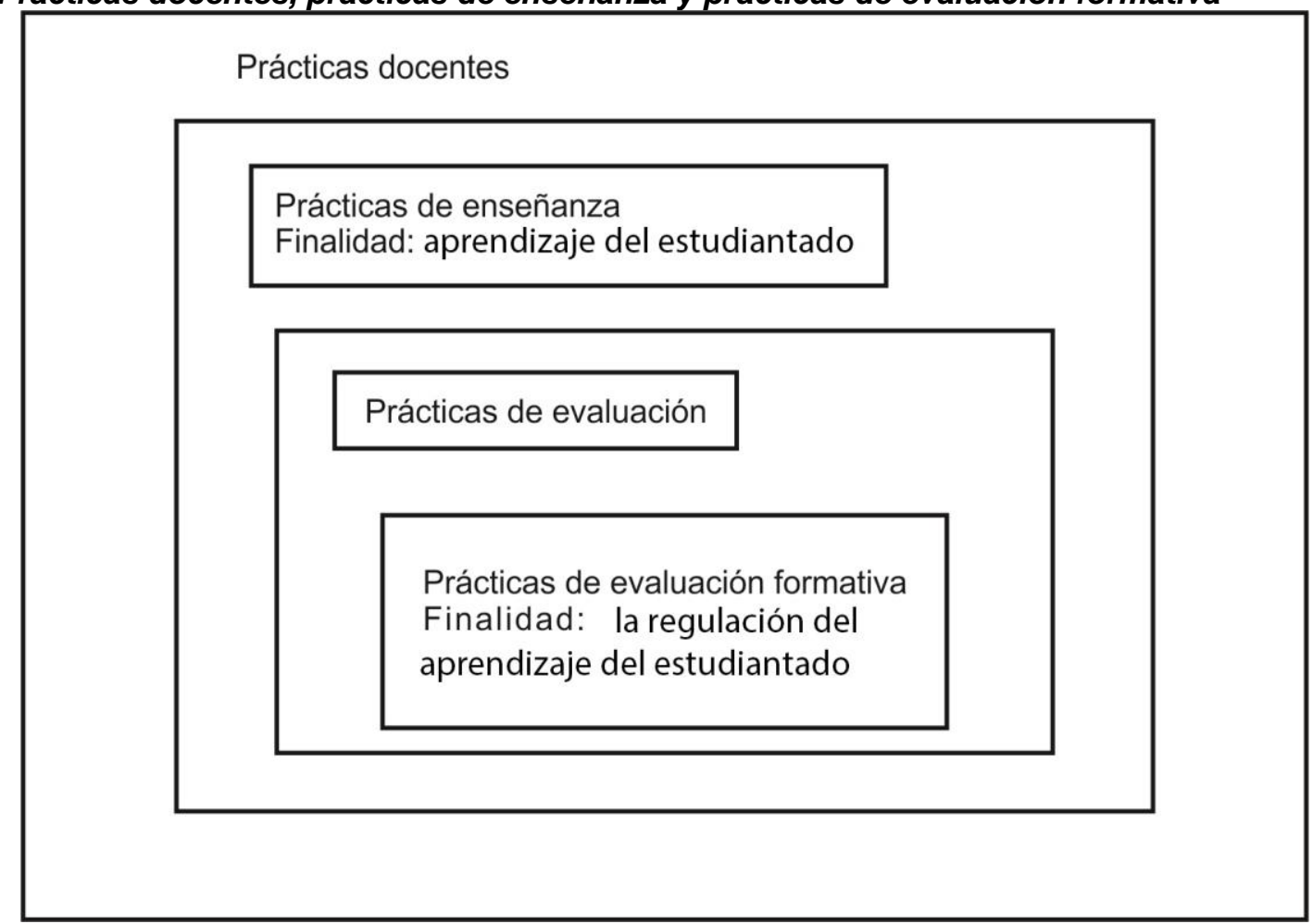

Fuente: Elaboración propia, 2017

\subsection{Una definición de las prácticas de evaluación formativa}

La revisión de las prácticas de evaluación formativa requiere de una definición de este concepto, de las precisiones sobre los conceptos centrales de regulación del aprendizaje y de situación auténtica, y una aclaración sobre el lugar que las prácticas de evaluación formativa ocupan en las prácticas de enseñanza.

Allal y Mottier-Lopez (2005) muestran la evolución del concepto de evaluación formativa desde la concepción inicial neobehaviorista de Bloom. En el origen, bajo el control 
del profesorado y teniendo lugar exclusivo al final de una secuencia de aprendizaje, la evaluación formativa, en su concepción ampliada, se vive en todo momento del proceso de aprendizaje y da un papel activo al estudiantado.

Esta visión enriquecida de la evaluación formativa ha llevado a Allal (1988) a distinguir diferentes tipos de regulación externa, esencialmente en función del momento en que tiene lugar. La regulación proactiva ocurre cuando la recopilación de la información permite ajustar la enseñanza, al comienzo del proceso de enseñanza y aprendizaje, con respecto a un objeto dado a fin de tener en cuenta las diferencias entre el alumnado. La regulación interactiva interviene en el curso de las interacciones entre el estudiantado y los componentes de la situación (el grupo de pares, docente, material que favorece la autorregulación, etc.), lo que permite las adaptaciones continuas durante el proceso de aprendizaje (adaptación de los objetivos, criterios, herramientas, etc.). La regulación se considera retroactiva cuando se realiza al final de una secuencia de enseñanza y aprendizaje para identificar los aprendizajes logrados o no por el estudiantado. Implica la selección de medios y enfoques para corregir o superar las dificultades de aprendizaje (Allal y Mottier-Lopez, 2005).

A partir del trabajo de Black y Wiliam (1998), en esta investigación, la evaluación formativa refiere al proceso cíclico mediante el cual el alumnado o sus docentes realizan la recopilación (toma y registro) y el procesamiento (análisis e interpretación) de la información para emitir un juicio sobre el aprendizaje. La información recopilada se puede utilizar como retroalimentación para que cada estudiante active los procesos internos y autorregule sus aprendizajes. La información da razón sobre la adecuación de la enseñanza en relación con los diferentes estudiantes; entonces, ésta puede guiar a cada docente para regular su propia acción. La evaluación formativa puede ser espontánea e informal o planificada o formal. Además, una perspectiva constructivista resalta el hecho de que la regulación del aprendizaje solo la puede lograr el estudiantado, mientras que el profesorado solo puede intervenir indirectamente en esta regulación (representada por la línea punteada en la Figura 2). Como propone Tardif (2006), se distinguirá la regulación externa o heterorregulación de la regulación interna o autorregulación. 
Figura 2

El ciclo de la evaluación formativa

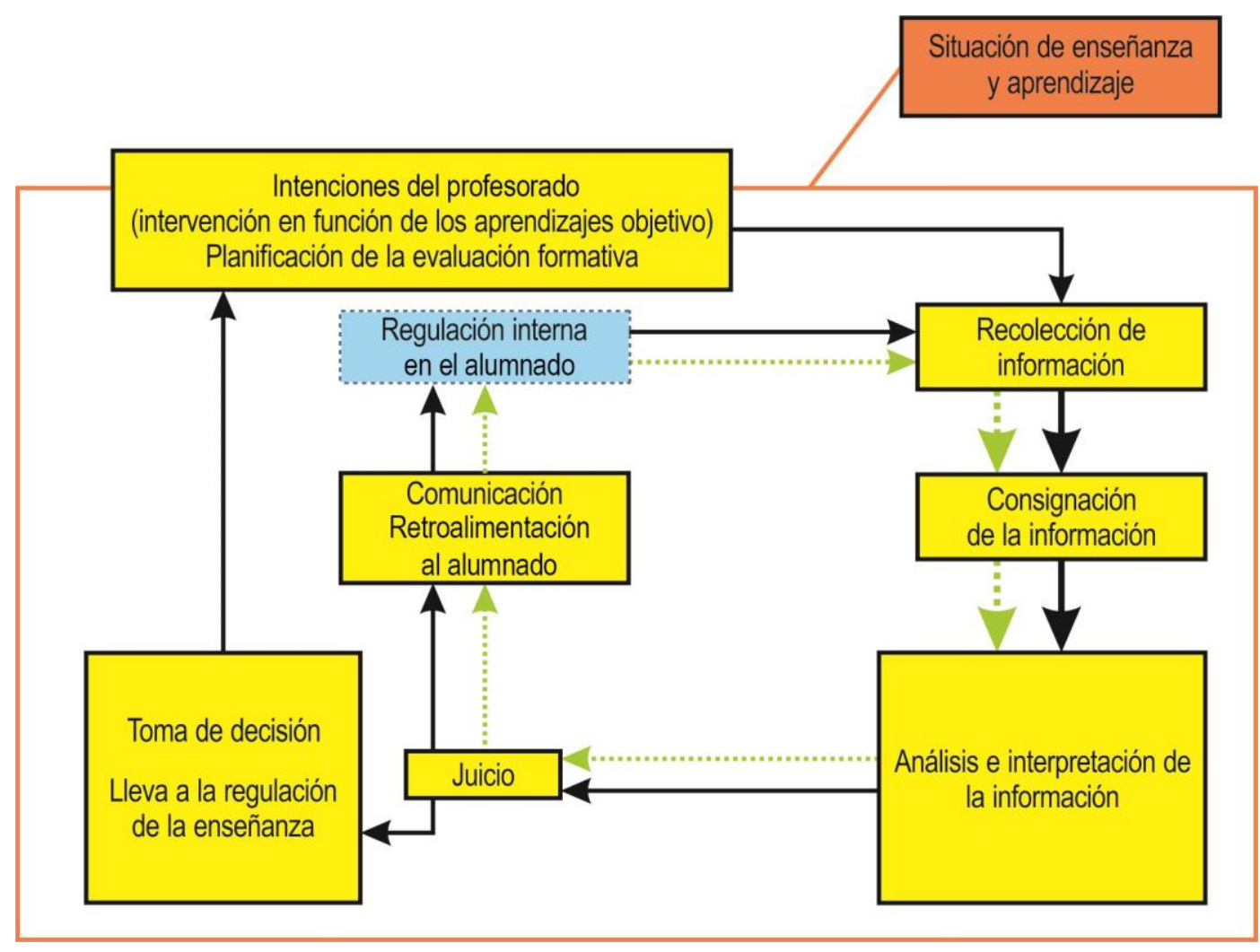

Fuente: Elaboración propia, 2017

Entre los conceptos importantes presentados por diferentes autores, quienes han tratado la evaluación formativa, se retoman los de situación auténtica y regulación del aprendizaje. Según Wiggins (1998), una situación auténtica se caracteriza por el hecho de que puede ser experimentada en la vida real; que exige juicio e innovación del estudiantado; que reproduce o simula las características de un contexto real de acuerdo con los objetivos, roles, situaciones, ambigüedades y limitaciones; que evalúa la capacidad del alumnado para utilizar un repertorio de conocimientos y saberes técnicos; que brinda oportunidades para practicar y consultar recursos; y, por último, que permite recibir retroalimentación.

En cuanto a la noción de regulación del aprendizaje, ha sido tratada, en la última década, desde el ángulo de la autorregulación del aprendizaje, concepto consistente con la perspectiva constructivista, que se conserva aquí. Varios modelos, como los de Butler (2006) y Zimmerman (2005) permiten su estudio, pero considerando el objetivo de esta investigación, se han conservado los trabajos que tratan las intervenciones o las prácticas que apoyan la autorregulación del aprendizaje del estudiantado. Perry, VandeKamp, Mercer 
y Nordby (2010), quienes analizaron las interacciones entre el profesorado y el estudiantado para apoyar la autorregulación del aprendizaje, establecieron las características siguientes: dar a los diferentes estudiantes las oportunidades para tomar decisiones, controlar los desafíos que se les presentan, evaluar sus producciones y las de sus pares, proporcionar apoyo a los diferentes estudiantes en los aspectos transferibles de una situación de aprendizaje a otra, garantizar que la evaluación formativa se desarrolle en un clima no amenazante y que las intervenciones estén orientadas hacia el dominio del aprendizaje más que solo al desempeño.

Así, como se muestra en la Figura 2, las prácticas de enseñanza basadas en la evaluación formativa constituyen un subconjunto de las prácticas de evaluación definido como el conjunto de los actos singulares de cada profesional, así como los significados que este último da a las fases preactiva, interactiva y postactiva. Estos actos, observables o no, tienen por finalidad la regulación del aprendizaje por cada estudiante (autorregulación) mediante la regulación de la enseñanza. Estas acciones incluyen la recopilación y el procesamiento de la información sobre los aprendizajes de los diferentes estudiantes, un juicio sobre estos aprendizajes y una retroalimentación al estudiante para apoyar su autorregulación. Sobre la base de Bru (2013), se presentan las variables que se deben tener en cuenta al estudiar las prácticas de evaluación formativa, agrupándolas en tres conjuntos: las variables de proceso (dinámica de la comunicación, roles del profesorado y del estudiantado, dinámica del aprendizaje, procesos de regulación externa o interna), las variables relacionadas con los dispositivos (herramientas utilizadas, organización del tiempo y del espacio) y las variables vinculadas con los contenidos (elección de los objetos de aprendizaje y de las actividades, y la estructuración de los contenidos).

El objetivo de la presente investigación ha sido entonces caracterizar las prácticas de evaluación formativa del profesorado de primaria de acuerdo con las tres categorías de variables enumeradas anteriormente.

\section{Metodología}

\subsection{Enfoque}

En esta investigación, se ha empleado el enfoque cualitativo en el campo de las prácticas de evaluación formativa del profesorado de educación primaria, y se han adoptado los diseños tanto etnográfico como estudios de casos para abordar las interacciones del profesorado y del estudiantado en un conjunto de actividades de evaluación. El alcance es 
descriptivo e interpretativo, por cuanto se especifica la dimensión observable de las prácticas de evaluación formativa y se interpreta la dimensión inobservable de tales prácticas, es decir, los actos mentales de cada docente.

\subsection{Participantes}

La presente investigación ha comprendido al profesorado seleccionado en forma intencional a conveniencia de los ciclos III, IV y V de la educación primaria en la institución educativa Corazón de Jesús de la ciudad de Puno- Perú. Los criterios de inclusión para tener parte en la investigación han sido los siguientes: el que sean docentes que hayan incorporado las características de la evaluación prescritas por la renovación pedagógica, así como que manifestaran voluntad en participar. Esta investigación se desarrolló el segundo semestre del año 2017.

De acuerdo con los criterios de inclusión definidos en el presente estudio, trece docentes de educación primaria, reconocidos en su entorno por sus prácticas de evaluación correspondientes a los requerimientos del Ministerio de Educación del Perú, vía firma de consentimiento, decidieron libremente participar. Entre quienes participaron, cinco trabajaban en el tercer ciclo, dos en el cuarto ciclo y seis en el quinto ciclo. Dos docentes tenían más de veinte años de experiencia; siete tenían entre once y veinte años de experiencia; otros tres, entre seis y diez años; y solo una persona comenzó la carrera hace menos de seis años.

Se observó un conjunto de 25 actividades de aprendizaje y enseñanza. Estas actividades, que duraron aproximadamente 50 minutos, son parte de una secuencia, definida como el conjunto de actividades de enseñanza centradas en un aprendizaje precisado por el personal docente. Cada una de las 25 actividades centradas en la evaluación formativa fue elegida por cada docente en un área curricular de su elección.

\subsection{Técnicas de recopilación}

Los datos fueron recolectados durante dos entrevistas y una observación filmada. La recopilación de datos comenzó con una entrevista estructurada abierta (Patton, 2002) que permitió recopilar datos, principalmente, sobre la planificación de la actividad elegida (objeto, proceso y herramientas de evaluación formativa), sobre su lugar en la secuencia más amplia de aprendizaje - enseñanza concerniente a un tema determinado, así como sobre las concepciones de aprendizaje, enseñanza y evaluación formativa. 
Luego, la actividad en clase fue observada y filmada, mientras se enfocaban las acciones de cada docente en los planos visual y sonoro. También, dos micrófonos permitieron captar las intervenciones de cualquier estudiante.

Finalmente, esta observación filmada fue seguida por una entrevista de recuerdo estimulado (Henderson y Tallman, 2006), que aprovechó el tipo de preguntas de estímulo de Vermersch (2010); a saber, las preguntas de inicialización, focalización, elucidación y de regulación de la entrevista. La entrevista de recuerdo estimulado fue realizada a partir de la visualización de segmentos del video de la actividad observada, considerados significativos en el plano de la evaluación formativa. La mitad de estos segmentos fue elegida por cada docente y la otra por el equipo de investigación. El conjunto de los extractos duró aproximadamente cuatro minutos. Durante la entrevista, cada docente fue invitado a interrumpir la visualización para nombrar lo que era observable en la cinta de video y verbalizar sus acciones mentales concomitantes.

\subsection{Procesamiento de análisis}

Los datos recopilados se procesaron en dos aspectos, el primero proporcionó un análisis preciso y el segundo permitió un análisis más global. Este último fue seleccionado para los fines del presente artículo. Esta selección, que impuso límites importantes en el examen de los procesos, ha llevado a conservar algunos indicadores que abandonaron su aspecto dinámico. El enfoque utilizado en este nivel de análisis se inspira en Bru (2013) y Paquay (2004). A partir de los datos de la entrevista estructurada abierta y de la observación filmada, la ficha de análisis global, presentada en el Anexo 1, tomó en cuenta las variables de proceso (ítems 1 y 5), las variables relacionadas con los dispositivos (ítems 2, 3, 4 y 8) y las variables relativas al contenido (ítems 6 y 7). Así, la ficha de análisis global comprende los ítems siguientes:

- La temporalidad de la evaluación formativa (micro o macro) (ítem 1): cuando la actividad de evaluación formativa se registró en el largo tiempo fue codificada macro, incluso si ésta comprendió también los ciclos de evaluación formativa, que caracterizan el corto tiempo, mientras que otra que solo se situó en el corto tiempo fue codificada micro.

- El rol del profesorado en la actividad (ítem 2): en función de hacer uso o no de los instrumentos de recopilación y procesamiento de la información, de juicio o de retroalimentación, y en función de la concepción de estas herramientas realizada o no por el personal docente (ítem 3). 
- Las modalidades de organización del trabajo del estudiantado (ítem 4): observadas en los diferentes momentos de la actividad: grupo de clase, pequeños equipos o contacto individual.

- La función de la evaluación formativa (ítem 5): observada al considerar las regulaciones retroactiva, interactiva y proactiva en la secuencia descrita por cada docente.

- El tipo de actividad con respecto a los objetos de aprendizaje (ítem 6): se distinguieron seis tipos potenciales a partir del cruce entre el carácter auténtico o no de la actividad y tres objetos de aprendizaje posibles (competencia, componentes, conocimientos esenciales).

- Los objetos de evaluación formativa en función de los dominios del aprendizaje (ítem 7): competencias transversales o disciplinares, componentes de estas y conocimientos esenciales.

- El rol del estudiantado (ítem 8): en función de la asunción del estudiantado de una u otra de las etapas del proceso de evaluación formativa, el rol mínimo se refiere a una participación específica más que hacerse cargo.

El análisis global se centró en la autorregulación del aprendizaje a través del examen de ciertas características de la actividad susceptibles de apoyar esta autorregulación. A partir de los datos de observación, se trató en relación con el estudiantado, su función (control de los desafíos, autoevaluación y evaluación entre pares durante o al final de la tarea) y las características de la tarea propuesta (apertura de la tarea, importancia de las interacciones sociales para tener éxito en la tarea y la práctica de acciones de autorregulación). También, se abordó en relación con el profesorado, su rol (modelado cognitivo, solicitud de verbalización de sus procesos a cualquier estudiante, proposición o recordatorio de objetivos y criterios de evaluación formativa).

\section{Resultados}

Los resultados son presentados en función de cada una de las tres categorías de variables enumeradas previamente: procesos, dispositivos y contenidos. Para cada una de estas categorías, primero se informan los resultados del conjunto de las actividades estudiadas y luego se procede a realizar un análisis basado en los ciclos y en las áreas de aprendizaje. 


\subsection{Variables de procesos: temporalidad de la evaluación, roles del profesorado y del estudiantado, y tipo de regulación}

El análisis global realizado en el conjunto de las actividades muestra que, en todos los casos, la evaluación formativa se hizo dentro de las interacciones espontáneas del profesorado y el alumnado a través de las cuales se produjeron las microsecuencias de "recolección-procesamiento-juicio-retroalimentación". En la mitad de los casos, la evaluación solo se realizó en el corto tiempo (12/25) (ver síntesis del análisis global en el Anexo 1, ítem 1 - micro), mientras que en la otra mitad, se situó tanto en el corto tiempo como en el largo tiempo (13/25, ítem 1 - macro). En cuanto a las herramientas, de las doce actividades que solo se situaron en el corto tiempo, diez no utilizaron herramientas específicas para la evaluación formativa (se volverá a las herramientas en la sección sobre los dispositivos). Esta situación se invierte en las actividades ubicadas en el largo tiempo (macro), ya que en nueve de los trece casos se utilizaron herramientas diseñadas para fines de la evaluación formativa (actividades calificadas como instrumentadas).

En cuanto al rol del estudiantado, en más del $50 \%$ de las actividades (14/25, ver Anexo 1, Ítem 7), el personal docente no le confirió ningún rol o un papel mínimo en una u otra de las etapas del proceso de evaluación formativa. En casi una cuarta parte de las actividades (6/25), el profesorado dio un rol más activo al estudiantado, que lo condujo a apropiarse de las herramientas de evaluación formativa, como una guía de evaluación del proceso de resolución de problemas o una guía de evaluación del trabajo de cooperación en un equipo de trabajo. Por lo tanto, se puede decir que, a menudo, diferentes estudiantes participaron en el proceso evaluativo sin asumir un rol, el cual estuvo a cargo exclusivamente del profesorado.

En relación con la regulación externa (Allal, 1988), que puede ejercer cualquier docente, el análisis de los datos (ver el Anexo 1, ítem 4) indicó que la mayoría de las actividades observadas (17/25) favoreció una regulación externa interactiva, que la regulación retroactiva estuvo presente en siete actividades y que una actividad privilegió la regulación proactiva.

En cuanto a la regulación interna, se examinó desde el punto de vista de las características de las actividades susceptibles de apoyar la autorregulación del aprendizaje del estudiantado (características de la tarea y rol de cada estudiante) y del rol del profesorado. Todas las actividades observadas comprendieron una presentación o un recordatorio de las estrategias de aprendizaje (25/25), y en casi la totalidad (23/25), el 
personal docente propuso o recordó los objetivos y los criterios de evaluación formativa. El modelado cognitivo estuvo presente en una quinta parte de las actividades (5/25). En cuanto a las características de la actividad, y, más específicamente, de las características de la tarea, una pequeña proporción de las situaciones observadas fueron estructuradas alrededor de una tarea abierta (5/25) o llevaron al estudiantado a ejercitarse en la utilización de estrategias de autorregulación del aprendizaje (4/25). En relación con el rol del estudiantado, pocas actividades ofrecieron oportunidades de autoevaluación durante la secuencia de aprendizaje (3/25) o de evaluación entre pares (3/25).

\subsection{Variables relativas a los dispositivos: instrumentos y modalidades de gestión del trabajo del estudiantado}

Como se muestra en el Anexo 1, en la mayoría de los casos en los que la evaluación formativa solo se realizó en las microsecuencias, que caracterizan el corto tiempo de la referida evaluación, no se utilizó ningún instrumento conocido específicamente para los fines de tal evaluación. Estas actividades han sido clasificadas como no instrumentadas en el plan de la evaluación (10/12). Por el contrario, en la mayoría de los casos, en los que la evaluación formativa se hizo en el largo tiempo, cada docente recurrió a los instrumentos (9/13) que diseñaron (por ejemplo, las guías de autoevaluación).

Con respecto a la organización del trabajo del estudiantado, surgieron tres modalidades durante el desarrollo de las actividades (Ver Anexo 1, ítem 3): el profesorado intervino ante una o un estudiante en presencia o no del grupo de clase (15/25), ante los subgrupos de estudiantes (9/25) o ante el grupo de la clase (7/25). El total de los números indica que en cuatro casos, dos modalidades alternaron en la misma actividad: el profesorado intervino ante una o un estudiante y delante del grupo de clase o intervino frente a pequeños equipos de trabajo y ante el grupo de clase.

\subsection{Variables relativas a los contenidos: elección de los objetos de aprendizaje y tipos de actividades}

Un primer examen indica que trece de las actividades analizadas corresponden al aprendizaje de la lengua; nueve, a matemáticas y tres conciernen a otras áreas (el área de personal social o el de la ciencia y tecnología). En cuanto a los objetos de aprendizaje y de evaluación formativa, de acuerdo con la síntesis del análisis global (Anexo 1, ítem 6), se obtuvieron los resultados que muestran que las actividades se enfocaron en los 
conocimientos esenciales (15/25) y que los componentes de las competencias (12/25) predominaron. Con respecto al tipo de actividades (Anexo 1, ítem 5), veintidós de las veinticinco actividades analizadas pueden ser calificadas como no auténticas.

\subsection{Algunos ejemplos de prácticas extraídas de tres estudios de casos}

Si el análisis global, que muestra los resultados presentados anteriormente, proporciona información sobre la dimensión observable de las prácticas de evaluación formativa, los estudios de casos, que aprovecharon los datos recopilados mediante las entrevistas de recuerdo estimulado, permiten acceder a la parte inobservable de las prácticas de enseñanza, es decir, a los actos mentales de cada docente. Los ejemplos seleccionados revelan los modos de organización de la situación de evaluación o las modalidades de utilización del material, que fueron particularmente interesantes.

\subsubsection{Un día bien ocupado: Rocío}

Rocío propuso actividades significativas que encajaron en un marco temporal adaptado al estudiantado del tercer ciclo, es decir, una jornada escolar. Por la mañana, Rocío invitó a sus estudiantes a elegir una foto y a escribir dos o tres oraciones para contarlas. Rocío corrigió durante la pausa del medio día y agrupó las producciones del estudiantado según el tipo de dificultades: cronología, estructura de las oraciones, empleo de la letra mayúscula y el punto. Por la tarde, los equipos de trabajo fueron constituidos de acuerdo con tales dificultades. Cada estudiante corrigió su texto solo o con la ayuda de sus pares. Luego, Rocío invitó a algunos estudiantes a explicar las correcciones que hicieron y aprovechó la oportunidad para hacer un repaso con tales estudiantes en el grupo de clase.

Los procesos de evaluación formativa observados en Rocío se actualizaron tanto en los macroprocesos, que se desarrollaron a lo largo del día, como en los micros procesos, que caracterizaron las interacciones espontáneas de Rocío con sus estudiantes agrupados en equipos. Además, el análisis de sus comentarios proporcionó información sobre los procesos de procesamiento de la información a nivel micro, en los que Rocío se dedicó a la interacción. Este análisis muestra una consideración del contexto e historicidad (momento del año escolar y duración del trabajo de los diferentes estudiantes), del nivel de dificultad del objeto de aprendizaje y de las características cognitivas y afectivas de cada estudiante. Rocío prestó especial atención al aspecto no verbal del estudiantado: intentó buscar en sus ojos para ver si había un clic. Las palabras de Rocío indicaron también que, la 
representación que ella tenía de sus estudiantes se construyó continuamente a través de sus interacciones.

Finalmente, los comentarios de Rocío proporcionaron una luz interesante sobre los vínculos entre los macroprocesos y microprocesos. Las interacciones observadas entre Rocío y sus estudiantes dieron testimonio de microprocesos de evaluación formativa marcados por la espontaneidad. Sin embargo, según Rocío, estos microprocesos comprendieron un aspecto que se planificó bajo la influencia de los macroprocesos de evaluación formativa también presentes en esta actividad. Estos microprocesos, que atestiguan las interacciones de Rocío, ganaron precisión al enfocarse en ciertas dimensiones del aprendizaje del estudiantado a la luz de las intenciones de la evaluación formativa establecidas durante la planificación de los macroprocesos.

\subsubsection{Una reunión privilegiada para una evaluación compartida: Luis}

Luis hizo que sus estudiantes del segundo ciclo autoevaluaran su lectura en voz alta en reuniones individuales. El estudiantado permitió que Luis realizara una coevaluación. Luis creó una organización particular que le permitió intervenir individualmente ante cada uno de sus estudiantes durante unos treinta minutos, en ausencia del resto del estudiantado de la clase. El estudio de caso destaca la organización que hizo posible esta reunión privilegiada, así como los subprocesos de procesamiento, juicio y toma de decisiones inherentes a esta coevaluación.

Para Luis, a fin de que cada estudiante se convierta en un mejor lector, era importante que se diera cuenta de su nivel de dominio de los diferentes componentes de la lectura en voz alta. Gracias a la colaboración de una colega, el alumnado tuvo, unos días antes de la reunión con Luis, grabada su lectura de un texto. Para que esta reunión se llevara a cabo, Luis utilizó dos modos de organización: se reunió con el alumnado durante el período del almuerzo o después de la clase. Para compensar el tiempo dedicado por Luis a estas reuniones fuera del horario de clases, la dirección de la escuela acordó ajustar el horario de la escuela para que estas reuniones reemplacen el tiempo de supervisión.

Los comentarios de Luis indicaron claramente los subprocesos de procesamiento, juicio y toma de decisiones inherentes a esta coevaluación. Luis se dio cuenta de que estaba haciendo el mismo proceso de evaluación mental que el que le pidió a cada estudiante. Mientras hacía esta evaluación mentalmente, Luis se preguntaba sobre la retroalimentación que les iba dar al estudiantado. Esta tuvo una doble finalidad: comunicar el fruto de su propia 
evaluación y dar las indicaciones al alumnado para que él o ella desarrolle su capacidad de autoevaluarse. Luis constató que lo que anotó en la guía constituía también una comunicación para los padres, ya que esta guía completada se agregó al portafolio de cada estudiante.

Además, Luis recordó que aunque todo su proceso de evaluación se apoyó en una norma individual, "cada niño fue quien se enfrentó a sí mismo, no se comparó con el resto". Luis destacó el hecho de que la norma individual se reconsideró en la escala de evaluación del grupo de clase: "entonces, estoy seguro que me posicioné en relación a lo que se ha trabajado, como se ha trabajado, a donde espero que el estudiantado debe ser capaz de llegar en esta época del año". En esta situación precisa de coevaluación de la lectura de cualquier estudiante, la interpretación de los criterios y el juicio estuvieron estrechamente relacionados con toda la experiencia del grupo en los últimos meses.

\subsubsection{Hacer manipular el material para observar los procesos: Gabriela}

Al examinar el cuaderno de los diferentes estudiantes de su clase del segundo ciclo, Gabriela constató que el alumnado tenía dificultades con los números de 5 o 6 dígitos. Ella comenzó la actividad con la intención de prestar una atención particular a Pablo. Gabriela pidió a sus estudiantes resolver diversas operaciones en el ábaco a fin de verificar su comprensión de las cuatro operaciones con los números y su dominio del ábaco. Ella confío en el trabajo de equipo para que el estudiantado se ayudara en esta tarea. El caso de Gabriela permitió identificar el aporte del material de aprendizaje, específicamente de un material de manipulación en matemáticas, al tomar información sobre los procesos mentales del estudiantado. Este caso posibilitó igualmente ejemplificar un proceso de orientación que ayudó a un estudiante en dificultades.

El análisis de las retroalimentaciones de Gabriela mostró que cuando ésta intervino en los procesos de aprendizaje, ella lo hizo guiando al estudiantado paso a paso. Este tipo de intervención apareció precisamente en el caso de Pablo, quien no estaba familiarizado con el ábaco, pero que logró utilizarlo con éxito gracias a las intervenciones de Gabriela. También, fue interesante notar que la descripción de la acción de Pablo fue asumida completamente por Gabriela: Pablo respondió a las preguntas de Gabriela manipulando el material. Entonces, se puede suponer que el material de aprendizaje le ayudó a Pablo a pasar una primera etapa en la comprensión, en la que pudo experimentar dificultades en describir lo que hacía mentalmente. Por lo tanto, se cree que el material de aprendizaje jugó un triple rol: 
le facilitó a Gabriela la observación de los procesos implementados por Pablo y del inicio del proceso de evaluación formativa, al mismo tiempo que favoreció el trabajo de Pablo sobre las operaciones matemáticas y la conciencia de su enfoque.

\subsection{Discusión}

Para cada una de las categorías de variables, se retoma lo esencial de los resultados, se destaca su contribución al estudio de las prácticas de evaluación formativa y se proporcionan las pistas de interpretación.

Con respecto a las variables de procesos, los resultados mencionados anteriormente, que surgieron del análisis global o de los estudios de casos, contribuyen a sostener la pertinencia de la distinción entre dos marcos temporales de la evaluación formativa. En efecto, el análisis permite constatar que las microsecuencias de evaluación formativa, que caracterizan el corto tiempo, están presentes en todas las actividades y que a ellas se añaden las macrosecuencias. De manera interesante, el caso de Rocío puso en evidencia la influencia de los macroprocesos de evaluación formativa en los microprocesos.

Además, el estudio muestra que la evaluación formativa tiene principalmente una función de regulación externa interactiva. Las prácticas estudiadas dan testimonio de una concepción más amplia de la evaluación formativa, tal como la presentan Allal y MottierLopez (2005), y de dos maneras. En efecto, las micro secuencias contribuyen a una integración continua de la evaluación formativa en las actividades de aprendizaje y enseñanza experimentadas en la clase. Por otro lado, la función de regulación interactiva privilegiada permite constatar que cuando la actividad de evaluación formativa se registra en el largo tiempo, ella se sitúa en el curso del aprendizaje más que al final como la concepción inicial de la evaluación formativa lo aconsejaba.

Por otro lado, la intervención de los diferentes profesores se caracteriza en el conjunto de las actividades por las intervenciones de sugerencia o recordatorio de estrategias de aprendizaje, objetivos y criterios de la evaluación formativa. Aunque el estudio de casos de Luis proporciona un buen ejemplo de actividad dirigida a desarrollar la autoevaluación del estudiantado, el personal docente parece menos inclinado a recurrir a la autoevaluación y a la evaluación por los pares, y propone pocas ocasiones donde los diferentes estudiantes puedan implementar las estrategias de autorregulación. Este hecho se puede explicar por la novedad del concepto para el profesorado y por la polisemia de este último, como lo señala Tardif (2006). 
Con respecto a las variables relativas a los dispositivos, los resultados presentados indican menos presencia de una instrumentación específica de la evaluación formativa en el corto tiempo. Este resultado se puede explicar por el hecho de que esta evaluación formativa está tan integrada al aprendizaje y a la enseñanza que, naturalmente, explota las herramientas. El caso de Gabriela, quien observa el uso del ábaco por el estudiantado, ilustra bien las múltiples funciones del material de aprendizaje. La evaluación formativa, que se registra en el largo tiempo, explota más las herramientas de recopilación y procesamiento de la información, así como las herramientas de retroalimentación que le son propias. Es razonable pensar que todas estas formas de evaluación formativa se sitúan en dos ejes que se refieren a dos objetivos que guían las intervenciones del personal docente: el aprendizaje y la evaluación. Finalmente, con respecto a las variables relativas a los contenidos, los dominios disciplinares elegidos por cada docente participante corresponden a las áreas consideradas básicas en el plan curricular: comunicación en español y matemáticas. Estas materias ocupan un lugar importante en la tarea del personal docente dado el número de horas asignadas cada semana. Ellas corresponden también a los dominios de aprendizaje que se han tratado más ampliamente en la renovación pedagógica, particularmente en la evaluación de los aprendizajes.

En cuanto a los objetos de evaluación, los resultados indican un énfasis en los conocimientos esenciales, lo cual se podría explicar de dos maneras. Primero, debido a que los objetos evaluados siguen siendo similares a los del programa de estudios anterior, donde el acento estaba en los conocimientos esenciales, el personal docente podría inclinarse a confiar en las prácticas anteriores bien dominadas para introducir gradualmente otras nuevas. Luego, desde otra perspectiva, las prácticas observadas revelan una de las características importantes de la evaluación formativa, que en el curso del aprendizaje, es importante interesarse en los conocimientos esenciales y en los componentes de las competencias.

El tipo de actividades presentadas, es decir, las actividades de carácter no auténtico, plantean preguntas interesantes: ¿se deben preferir las actividades auténticas en todo momento en un contexto de evaluación formativa o se deben elegir, en diferentes momentos del desarrollo de una competencia, las tareas que contienen diversos grados de autenticidad y de complejidad variable, según el objeto a evaluar?

Además, más allá del enfoque utilizado para el análisis de los datos, se constata que varios de los resultados ilustran las categorías que describen la ecología de la clase. Por 
tanto, se puede creer que estas categorías permiten también describir las prácticas de evaluación formativa en un nivel más fino que el de la clase, en términos de comportamientos observables o de actos mentales que constituyen estas prácticas. Así, se puede observar:

- la multiplicidad de los eventos que ocurren o las dimensiones que se deben tratar para hacer un juicio sobre el aprendizaje del alumnado (Rocío y Luis);

- la inmediatez de los datos verbales y no verbales recopilados cuando la evaluación formativa se sitúa en el corto tiempo;

- la rapidez con la que las microsecuencias de evaluación formativa se desarrollan (característica de la evaluación formativa que se registra en el corto tiempo);

- la visibilidad de la acción del personal docente que, al proporcionar una retroalimentación al estudiantado, sabe también que está en comunicación con los padres de cada estudiante, quienes examinarán, por ejemplo, la guía de autoevaluación y de coevaluación completada por el alumnado y el profesorado (Luis);

- la historicidad de los eventos que se deben considerar en el procesamiento de la información recopilada y el juicio emitido (Rocío).

\section{Conclusiones}

La presente investigación se centró en un análisis de las prácticas de evaluación formativa del profesorado de primaria en la institución educativa Corazón de Jesús de la ciudad de Puno - Perú con el fin de proporcionar los ejemplos de prácticas que integran las características susceptibles de apoyar el aprendizaje del estudiantado tal como aparecen en el enfoque de referencia sobre la evaluación de los aprendizajes.

En general, los resultados que surgen del análisis de las 25 actividades de aprendizaje y enseñanza sobre la evaluación formativa muestran que el personal docente que ha participado en esta investigación ha integrado en sus prácticas muchas de las características de la evaluación formativa recomendada por el Ministerio de Educación del Perú (2016). Las concepciones de evaluación formativa expresadas por el profesorado dan testimonio de una concepción más amplia de la referida evaluación, que no se sitúa únicamente al final del aprendizaje, sino también durante el aprendizaje, de manera continua.

En relación con las prácticas de evaluación formativa del profesorado, en coherencia con sus concepciones, todas las prácticas observadas incluyen los microprocesos de recolección-procesamiento-juicio-retroalimentación, inscritos en el corto tiempo. La 
observación, la escucha y la memoria sostienen estos microprocesos. En la mitad de las actividades analizadas, se añaden también las prácticas que se registran en el largo tiempo de la evaluación formativa: se observa, la mayoría de las veces, la autoevaluación, la evaluación por los pares y la coevaluación a partir de guías diseñadas para este fin. Esto significa que cuando las actividades se registran en el corto tiempo, el material de aprendizaje sirve a la vez a los fines del aprendizaje y de la evaluación formativa, mientras que cuando las actividades se inscriben en el largo tiempo, un material que sirve al proceso de recopilación-procesamiento-juicio-retroalimentación es utilizado.

El profesorado hizo ver las actividades donde los conocimientos esenciales y los componentes de las competencias en matemáticas y en español son abordados en el marco de situaciones no auténticas. En cuanto a los dispositivos, en muchos casos, el personal docente implementa las estrategias que les permite interactuar con el estudiantado o delante de equipos de trabajo.

Estas actividades hacen posible una regulación externa interactiva, que se desarrolla durante el proceso de aprendizaje. Las actividades poseen ciertas características susceptibles de apoyar la regulación del aprendizaje del estudiantado. Estas características se refieren principalmente al rol del profesorado. Todas las actividades llevan al personal docente a proponer o recordar las estrategias de aprendizaje; en casi la totalidad, el profesorado propone o recuerda los objetivos y los criterios de evaluación formativa. Por otro lado, una pequeña proporción de actividades sugiere una tarea abierta al estudiantado, brinda las oportunidades de autoevaluación o evaluación por los pares durante la actividad o las ocasiones de ejercitarse en la utilización de estrategias de autorregulación del aprendizaje. Pocas actividades llevan al profesorado a realizar un modelado cognitivo. Lo que caracteriza a los roles del profesorado y del alumnado es que este último participa en el proceso de evaluación bajo la responsabilidad casi exclusiva del personal docente.

Los datos de observación indican que el profesorado ha regulado ocasionalmente la enseñanza en acción; sus comentarios, recopilados durante las entrevistas de recuerdo estimulado, resaltan otras manifestaciones de regulación e informan sobre los procesos de reflexión que surgieron durante la acción o que continuaron después. Las reuniones de análisis de prácticas permitieron al profesorado participante continuar este trabajo de reflexión ya presente en el curso de acción y después de la acción.

Asimismo, respecto al análisis de las prácticas docentes, en el plano conceptual, esta investigación ha permitido ubicar las prácticas de evaluación formativa dentro de las 
prácticas docentes. También, ha llevado a precisar una definición de la evaluación formativa en una perspectiva constructivista, al mostrar que solo el estudiantado puede regular sus aprendizajes. Considerar las dimensiones observables y no observables de las prácticas docentes permite una descripción más detallada, que incluye los procesos de procesamiento de la información y de juicio desarrollados por el profesorado. Además, aunque evocadas en la documentación ministerial, las dos formas que adopta la evaluación formativa han sido aquí ampliamente descritas, al distinguir dos marcos de tiempo, el largo tiempo y el corto tiempo, que comprenden los macroprocesos y los microprocesos, respectivamente. Es sobre todo la evaluación formativa espontánea e informal que se destaca, incluso se valora, en esta investigación.

Sobre el plan de apoyo al análisis crítico del profesorado participante, la intervención implementada muestra la pertinencia de estrategias de análisis de prácticas realizadas más cerca de las realidades. Tres guías de lectura sobre el proceso de evaluación formativa, la retroalimentación y las características de las actividades susceptibles de apoyar la autorregulación del aprendizaje llevaron al profesorado a un primer distanciamiento.

Además, la descripción de estas prácticas sugiere implícitamente algunas pistas de formación continua. El paso importante dado por una parte del profesorado ha sido compartir con el estudiantado la responsabilidad del proceso de regulación a través de la creación de las condiciones para que el estudiantado autoevalúe y autorregule sus aprendizajes.

Finalmente, los límites de la investigación presentada siguen siendo importantes, sobre todo porque el proceso de implementación de las prácticas de evaluación formativa continúa. En este sentido, el retrato expuesto aquí se debe ver como parcial y puntual, puesto que, en un contexto de reforma, las prácticas de enseñanza están en constante evolución. Este retrato se revela también incompleto en la medida en que el presente estudio no intentó relacionar las prácticas de enseñanza y el desempeño del alumnado. Las investigaciones dirigidas a establecer vínculos entre ciertas características de la evaluación formativa y el desempeño del estudiantado según los dominios de aprendizaje son de mayor importancia. Además, la presente investigación solo se centró en las prácticas de evaluación formativa, mientras que la literatura relativa a la evaluación de los aprendizajes comprende otras dimensiones relacionadas con la evaluación sumativa, que convendría examinar. Otras investigaciones sobre las prácticas de evaluación son necesarias para comprender mejor este subconjunto y orientar mejor los temas de formación prioritarios del profesorado. 


\section{Referencias}

Allal, Linda. (1988). Vers un élargisement de la pedagogie de maitrise: processus de regulation interactive, retroactive et proactive. En Michael Huberman (dir.), Assurer la reussite des apprentissages scolaires? (pp. 86-126). Paris: Delachaux \& Niestle.

Allal, Linda y Mottier-Lopez, Lucie. (2005). L'évaluation formative de l'apprentissage: revue de publications en langue française. En Centre pour la recherche et l'innovation dans l'enseignement (dir.), L'évaluation formative, pour un meilleur apprentissage dans les classes secondaires (pp. 265-299). Paris: Editions OCDE.

Altet, Marguerite. (2002). Une démarche de recherche sur la pratique enseignante: L'analyse plurielle. Revue Française de Pedagogie, (138), 85-93. Recuperado de https://www.persee.fr/doc/rfp 0556-7807 2002 num 13812866

Altet, Marguerite. (2014). Les enseignants et leurs pratiques professionnelles. En Jacky Beillerot y Nicole Mosconi (dir.) Traité des sciences et des pratiques de l'éducation (pp. 291-303). Paris: Dunod. doi.org/10.3917/dunod.beill.2014.01.0291

Beillerot, Jacky y Mosconi, Nicole. (2014). Traité des sciences et des pratiques de l'éducation. Paris: Dunod.

Black, Paul y Wiliam, Dylan. (1998). Assessment and Classroom Learning. Assessment in Education, 5(1), 7-74. doi.org/10.1080/0969595980050102

Bru, Marc. (2013). L'enseignant, organisateur des conditions d'apprentissage. En Jean Houssaye (dir.), La pédagogie : une encyclopédie pour aujourd'hui (pp. 103-117). Paris: ESF.

Butler, Deborah L. (2006). L'autorégulation de l'apprentissage et la collaboration dans le développement professionnel des enseignants. Revue Des Sciences de l'éducation, 31(1), 55-78. doi.org/10.7202/012358ar

Casalfiore, Stefania. (2000). L'activité des enseignants en classe. Contribution à la compréhension de la réalité professionnelle des enseignants. Cahier de Recherche du GIRSEF, 6, 4-22. Recuperado de https://halshs.archives-ouvertes.fr/halshs00603919/document

Cohen, Elizabeth G., Lotan, Rachel A., Abram, Percy L., Scarloss, Beth A. and Schultz, Susan E. (2002). Can groups learn?. Teachers College Record, 104(6), 1045-1068.

Fletcher, Jack M., Foorman, Barbara R., Denton, Carolyn A. y Vaughn, Sharon. (2006). Scaling Research on Beginning Reading: Consensus and Conflict. En Mark A. Constas y Robert J. Sternberg (Eds.), Translating Theory and Research into Educational Practice: Developments in Content Domains, Large Scale Reform, and Intellectual Capacity (pp. 53-75). Mahwah, NJ: Lawrence Erlbaum.

Garet, Michael S., Porter, Andrew C., Desimone, Laura, Birman, Beatrice F. y Yoon, Kwang Suk. (2001). What makes professional development effective? Result from a national sample of teachers. American Education Research Journal, 38(4), 915-945. Recuperado de https://www.jstor.org/stable/3202507?seq=1\#page scan tab contents 
Hadji, Charles. (1999). L'Évaluation démystifiée: Mettre l'évaluation scolaire au service des apprentissages. Paris: ESF.

Henderson, Lyn y Tallman, Julie. (2006). Stimulated Recall and Mental Models: Tools for Teaching and Learning Computer Information Literacy. Lanham, MD: Scarecrow Press.

Kagan, Dona M. (1990). Ways of Evaluating Teacher Cognition: Inferences Concerning the Goldilocks Principle. Review of Educational Research, 60(3), 419-469. doi.org/10.3102/00346543060003419

Klenowski, Val. (1995). Studen self-evaluation process in student-centered teaching and learning contexts of Australia and England. Assessment in Education, 2(2), 145-163.

Ministerio de Educación del Perú. (2016). Currículo Nacional de la Educación Básica. Lima: MINDEDU. Recuperado de http://www.minedu.gob.pe/curriculo/pdf/curriculo-nacional$\underline{\text { de-la-educacion-basica.pdf }}$

Organización para la Cooperación y el Desarrollo Económico. (2014). Guía del Profesorado TALIS 2013: Estudio Internacional sobre enseñanza y aprendizaje (TALIS) OECD Publishing. Recuperado de https://www.oecd-ilibrary.org/education/guia-delprofesorado-talis-2013 9789264221932-es

Paquay, Léopold. (2004). L' évaluation des enseignants: tensions et enjeux. Paris: L'Harmattan.

Patton, Michael Quinn. (2002). Qualitative research \& evaluation methods (3a ed.) Thousand Oaks, CA: Sage Publications.

Perry, Nancy E., VandeKamp, Karen O., Mercer, Louise K. and Nordby, Carla J. (2010). Investigating teacher-student interactions that foster self-regulated learning. Educational Psychologist, 37(1), 5-15. doi.org/10.1207/S15326985EP3701_2

Picaroni, Beatriz. (2009). La evaluación en las aulas de primaria: usos formativos, calificaciones y comunicación con los padres. Uruguay: Universidad Católica del Uruguay y Grupo de Trabajo sobre Estándares y Evaluación [GTEE-PREAL]

Rosales, Carlos. (2003). Criterios para una evaluación formativa. Madrid: NARCEA, S.A. de ediciones Madrid.

Solé, Isabel. (2001). Evaluar lectura y escritura: algunas características de las prácticas de evaluación innovadoras. Lectura y Vida, Revista Latinoamericana, 22(4), 2-15. Recuperado de http://www.lecturayvida.fahce.unlp.edu.ar/numeros/a22n4/sumario

Tardif, Jacques. (2006). Une idée puissante, mais polysémique: l'autorégulation des apprentissages. Vie Pédagogique, 140, 45-51. Recuperado de http://collections.banq.qc.ca/ark:/52327/bs22578

Vermersch, Pierre. (2010). L'entretien d'explicitation (6a. ed.). Issy-les-Moulineaux: ESF. 
Wiggins, Grant. (1998). Educative assessment: designing assessments to inform and improve student performance. San Francisco, CA: Jossey-Bass.

Wiggins, Grant. (1992). Creating tests worth taking. Educational Leadership, 49, 26-33. Recuperado de http://www.ascd.org/ASCD/pdf/journals/ed lead/el 199205 wiggins.pdf

Wilson, Suzanne M. and Berne, Jennifer. (1999). Teacher learning and the acquisition of professional knowledge: An examination of research on contemporary professlonal development. Review of Research in Education, 24(1), 173-209. doi.org/10.3102/0091732x024001173

Windschitl, Mark. (2002). Framing constructivism in practice as the negotiation of dilemmas: An analysis of the conceptual, pedagogical, cultural, and political challenges facing teachers. Review of Educational Research, 72(2), 131-175. doi.org/10.3102/00346543072002131

Zimmerman, Barry J. (2005). Attaining Self - Regulation: A Social Cognitive Perspective. En Monique Boekaerts, Moshe Zeodmer y Paul R. Pintrich (Eds.), Handbook of SelfRegulation, (pp. 1 -39). San Diego, CA: Academic Press. 
Anexo 1

Ficha de síntesis del análisis global de las actividades de evaluación formativa

\begin{tabular}{|c|c|c|c|c|c|c|c|}
\hline \multirow{2}{*}{ 1. Tiempo } & Micro & 12 & \multicolumn{2}{|c|}{ Instrumentada } & 02 & No instrumentada & 10 \\
\hline & Macro & 13 & \multicolumn{2}{|c|}{ Instrumentada } & 09 & No instrumentada & 04 \\
\hline $\begin{array}{l}\text { 2. Instrumento de } \\
\text { evaluación }\end{array}$ & \multicolumn{3}{|c|}{$\begin{array}{l}\text { Instrumento desarrollado } \\
\text { por cualquier docente }\end{array}$} & 08 & \multicolumn{2}{|c|}{ Desarrollado por otro } & 03 \\
\hline \multirow{4}{*}{\begin{tabular}{ll} 
3. & \multicolumn{2}{c}{ Modalidades de } \\
organización del & del \\
trabajo & del \\
estudiantado &
\end{tabular}} & & Inicio & \multicolumn{2}{|c|}{$\begin{array}{l}\text { Desarrollo de la } \\
\text { actividad }\end{array}$} & \multicolumn{3}{|c|}{$\begin{array}{l}\text { Retroalimentación/ } \\
\text { objetivación }\end{array}$} \\
\hline & Individual & 05 & \multicolumn{2}{|c|}{15} & \multicolumn{3}{|c|}{05} \\
\hline & $\begin{array}{l}\text { Pequeños } \\
\text { equipos }\end{array}$ & & \multicolumn{2}{|c|}{09} & & & \\
\hline & $\begin{array}{l}\text { Grupo de } \\
\text { clase }\end{array}$ & 20 & \multicolumn{2}{|c|}{07} & \multicolumn{3}{|c|}{06} \\
\hline \multirow{2}{*}{\multicolumn{2}{|c|}{$\begin{array}{l}\text { 4. Función de la evaluación en la } \\
\text { secuencia de aprendizaje }\end{array}$}} & \multicolumn{2}{|c|}{$\begin{array}{c}\text { Regulación } \\
\text { proactiva }\end{array}$} & \multicolumn{2}{|c|}{$\begin{array}{c}\text { Regulación } \\
\text { interactiva }\end{array}$} & \multicolumn{2}{|c|}{ Regulación retroactiva } \\
\hline & & \multicolumn{2}{|c|}{01} & \multicolumn{2}{|c|}{17} & \multicolumn{2}{|l|}{07} \\
\hline \multirow{2}{*}{$\begin{array}{l}\text { 5. Tipo } \\
\text { actividad }\end{array}$} & Auténtica & \multicolumn{6}{|l|}{03} \\
\hline & $\begin{array}{l}\text { No } \\
\text { auténtica }\end{array}$ & \multicolumn{6}{|l|}{22} \\
\hline \multicolumn{2}{|c|}{$\begin{array}{l}\text { 6ominios del aprendizaje y } \\
\text { competencias transversales }\end{array}$} & \multicolumn{2}{|c|}{ Competencia } & \multicolumn{2}{|c|}{ Componente } & \multicolumn{2}{|c|}{ Conocimiento esencial } \\
\hline \multicolumn{2}{|c|}{ Lengua materna } & \multicolumn{2}{|l|}{01} & \multicolumn{2}{|l|}{07} & \multicolumn{2}{|l|}{06} \\
\hline \multicolumn{2}{|l|}{ Matemáticas } & \multicolumn{2}{|l|}{01} & \multicolumn{2}{|l|}{02} & 07 & \\
\hline Personal social & & & & & & 02 & \\
\hline Ciencia y tecnología & & & & 01 & & & \\
\hline Competencias transv & ersales & & & 02 & & & \\
\hline $\begin{array}{l}\text { 7. Rol de cualquie } \\
\text { evaluación de su a }\end{array}$ & $\begin{array}{l}r \text { estudiante } \\
\text { prendizaje } 0\end{array}$ & $\begin{array}{l}\text { en la } \\
\text { la de }\end{array}$ & $\begin{array}{l}\text { Ningún } \\
\text { mínime }\end{array}$ & rol c & rol & 14 & \\
\hline & & & $\begin{array}{l}\text { Rol no } \\
\text { (ejemp } \\
\text { cuestio }\end{array}$ & $\begin{array}{l}\text { instrume } \\
\text { lo: } \\
\text { namiento }\end{array}$ & tado & 05 & \\
\hline & & & $\begin{array}{l}\text { Rol } \\
\text { (ejemp } \\
\text { evalua }\end{array}$ & $\begin{array}{l}\text { instrume } \\
\text { lo: guía } \\
\text { ción) }\end{array}$ & $\begin{array}{r}\text { tado } \\
\text { de }\end{array}$ & 06 & \\
\hline Ciclo de enseñar & & & $3^{\circ}$ ciclo & & & 15 & \\
\hline & & & $4^{\circ}$ ciclo & & & 01 & \\
\hline & & & $5^{\circ}$ ciclo & & & 09 & \\
\hline
\end{tabular}


Revista indizada en

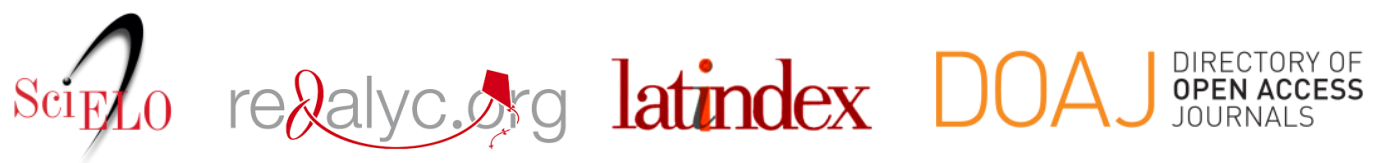

Distribuida en las bases de datos:

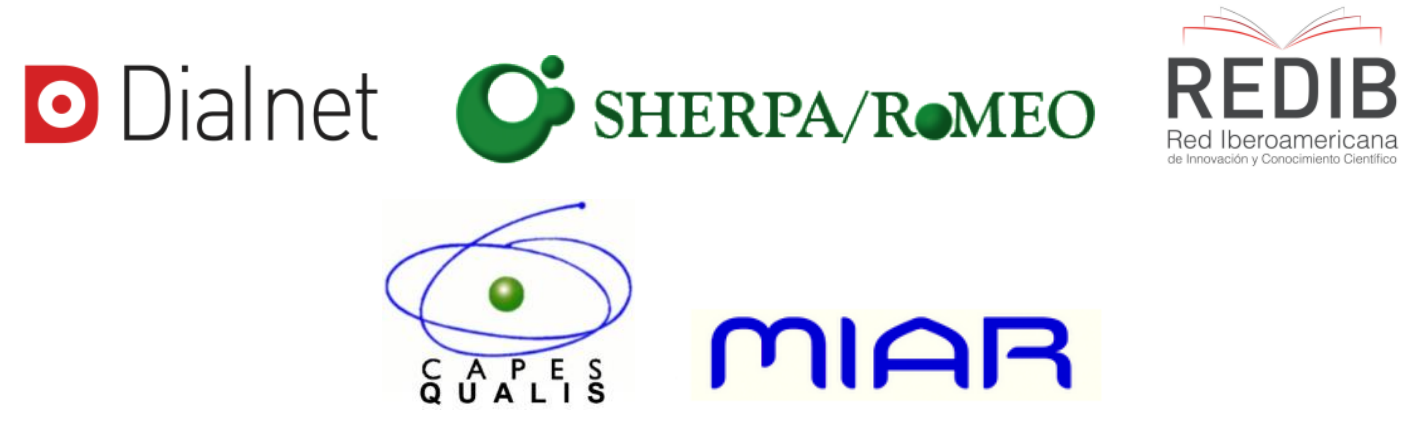

